\title{
Alterações cefalométricas presentes em crianças e adolescentes com desordens da ATM nas diferen- tes classificações sagitais de má oclusão
}

\author{
Leticia Vilaça Willeman Bastos*, Ricardo de Souza Tesch**, Odilon Victor Denardin***
}

\begin{abstract}
Resumo
Introdução: o acometimento da ATM por alterações degenerativas e deslocamentos do disco articular demonstrou ser um fator de risco para a identificação de indivíduos com padrões hiperdivergentes de crescimento facial. Objetivos: avaliar diferenças entre as médias encontradas para as variáveis cefalométricas em crianças com DTM articular e grupo controle, nas diferentes classificações sagitais de má oclusão (Classe I, II e III de Angle). Método: foram incluídos crianças e adolescentes com idade máxima de 17 anos, divididos em grupo experimental $(\mathrm{n}=30)$ com diagnóstico de DTM articular, segundo o eixo I do RDC/TMD para crianças e adolescentes, igualmente divididos em 3 sub-grupos $(n=10)$ de acordo com a classificação da má oclusão presente e grupo controle $(\mathrm{n}=30)$ sem DTM, pareados por gênero, índice de maturidade esquelética vertebral cervical e classificação da má oclusão. Foram traçadas telerradiografias laterais e as estruturas craniofaciais e suas relações foram divididas em: base do crânio, maxila, mandíbula, relações intermaxilares, relações esqueléticas verticais e relações dentárias. As diferenças encontradas entre as médias, para cada uma das variáveis, foram avaliadas pela aplicação do teste estatístico t de Student para amostra independentes. Resultados: o grupo experimental Classe I apresentou S.N.Ar $(\mathrm{p}=0,05)$ aumentado, o grupo experimental Classe II apresentou S.N.Ar $(p=0,006)$ e NperpA $(p=0,037)$ diminuídos e S.Ar. Go $(p=0,045)$ aumentado e o grupo experimental Classe III apresentou N.A.P ( $p=0,045)$, $1 . \mathrm{NB}(\mathrm{p}=0,001)$ e $1-\mathrm{NB}(\mathrm{p}=0,017)$ aumentados e $1.1(\mathrm{p}=0,004)$ diminuído, todos em relação aos seus respectivos controles Conclusão: as diferenças foram mais significantes nos pacientes com má oclusão de Classe II e de Classe III.
\end{abstract}

Palavras-chave: Crescimento facial. DTM. Má oclusão.

* Mestre em Ciências da Saúde - DTM e Dor Orofacial - Hosphel.

** Mestre em Ciências da Saúde - DTM e Dor Orofacial - Hosphel. Chefe do Setor de DTM e Dor Orofacial da Faculdade de Medicina de Petropólis.

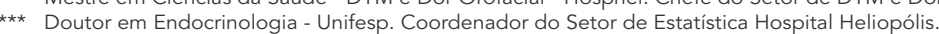




\section{INTRODUÇÃO}

As desordens temporomandibulares (DTM) podem ser definidas como um conjunto de condições dolorosas e/ou não funcionais, envolvendo os músculos da mastigação e/ou as articulações temporomandibulares (ATM) ${ }^{11}$. Apresentam prevalência de sintomas característicos, como dor e restrição de movimentos, entre 5\% e 15\%, com pico em adultos jovens, com idade entre 20 e 40 anos, preponderantemente do gênero feminino ${ }^{18}$. A prevalência de dor por DTM na infância e adolescência é menor do que a reportada em adultos jovens e situa-se entre $1 \%$ e $4 \%$, dependendo da idade $^{19}$

Atualmente, o Research Diagnostic Criteria for Temporomandibular Disorders (RDC/TMD) 8 , representa a ferramenta diagnóstica melhor investigada à cerca da validade e precisão para a classificação diagnóstica das DTM a partir de critérios diagnósticos específicos. Este sistema, através de seu eixo físico, permite a possibilidade de múltiplos diagnósticos para um mesmo indivíduo, sendo dividido em três amplas categorias não mutuamente excludentes: as dores miofasciais mastigatórias, com ou sem limitação de abertura de boca; os deslocamentos do disco articular, com ou sem redução; e as artralgias, osteoartrites e osteoartroses da articulação temporomandibular ${ }^{8}$.

O RDC/TMD não foi originalmente desenvolvido para ser aplicado em crianças e adolescentes, mas foi modificado e validado para este fim por Wahlund et al. ${ }^{29}$, tendo sido posteriormente aplicado neste grupo populacional por diversos pesquisadores ${ }^{19,29,30}$.

Estudos populacionais à cerca da prevalência dos sinais clínicos de DTM articulares, em crianças e adolescentes, demonstraram que $8 \%$ a $29 \%$ da população avaliada apresentam ruídos de estalido nas ATM, indicativos de deslocamento anterior do disco articular ${ }^{8}$, enquanto apenas $1 \%$ apresenta ruídos de crepitação ${ }^{25,10}$, indicativos de alterações degenerativas da $\mathrm{ATM}^{8}$.

$\mathrm{O}$ acometimento da ATM por alterações dege- nerativas e deslocamentos do disco articular demonstrou ser um fator de risco importante para a identificação de indivíduos com padrões hiperdivergentes de crescimento facial ${ }^{23,24}$. Contudo, os estudos que correlacionam DTM articulares e padrão de crescimento facial, em grande parte, o fazem utilizando pacientes adultos ${ }^{1,3,4,12,13,14,15}$.

De modo geral, os estudos acima citados não fizeram distinção entre os resultados encontrados em função da classe de má oclusão presente, tendo apenas um destes estudos avaliado exclusivamente pacientes com má oclusão de Classe $\mathrm{II}^{1}$ e outro avaliado pacientes com má oclusão de Classe II e III ${ }^{15}$.

Em artigo recém publicado, Hwang et al. ${ }^{15}$ avaliaram pacientes adultos com má oclusão de Classe I, Classe II e Classe III, classificados de acordo com as diferenças encontradas para o ângulo ANB, com diagnóstico de DTM e comparou-os com indivíduos assintomáticos, pareados por gênero e tipo de má oclusão apresentado. Todos os grupos de pacientes com diagnóstico de DTM apresentaram diminuição do comprimento do ramo mandibular. Os grupos Classe I e Classe II apresentaram ângulo goníaco aumentado e maior inclinação palatina dos incisivos superiores. Os grupos Classe II e Classe III apresentaram comprimento efetivo da mandíbula diminuído. Os pacientes do grupo Classe III apresentaram comprimento do corpo mandibular diminuído e inclinação do plano oclusal aumentada, ambos em relação à base do crânio.

Estas diferenças nos valores encontrados para as medidas cefalométricas de pacientes adultos com diferentes tipos de má oclusão (Classe I, II e III) e desordens da ATM, refletem, provavelmente, a influência do padrão distinto de crescimento mandibular observado nestes diferentes subgrupos diagnósticos. Assim, tais diferenças devem, hipoteticamente, ser possíveis de serem demonstradas em pacientes nos diferentes estágios de crescimento.

O objetivo do presente estudo foi avaliar dife- 
renças entre as variáveis cefalométricas selecionadas para a identificação do padrão de crescimento facial, em crianças com DTM articular e grupo controle livre da referida desordem, nas diferentes classificações sagitais de má oclusão (Classes I, II e IIII).

\section{MATERIAL E MÉTODOS}

O presente estudo seguiu protocolo observacional e transversal, realizado na Clínica de Ortodontia Preventiva da Faculdade de Medicina de Petrópolis-RJ, incluindo pacientes com idade máxima de 17 anos, de ambos os gêneros. Foi aprovado pelo Comitê Científico e de Ética na Pesquisa da Faculdade de Medicina de Petrópolis.

O grupo experimental foi composto de $30 \mathrm{pa}-$ cientes com diagnóstico de DTM articular, igualmente divididos em 3 sub-grupos $(n=10)$ de acordo com a má oclusão presente (grupos Classe I, II e III). Foram considerados critérios de inclusão: (1) a presença de DTM articular, grupo II e/ ou III, de acordo com o eixo I do RDC/TMD para crianças e adolescentes - versão para a língua portuguesa $^{26}$; (2) boa qualidade das telerradiografias laterais prévias ao tratamento ortodôntico, realizadas em um mesmo cefalostato (Clínica Radiológica Cerdo, Petrópolis, RJ). Como critérios de exclusão foram adotados: (1) diagnóstico de artrite reumatóide juvenil ou outras patologias articulares sistêmicas; (2) realização de tratamento ortodôntico prévio ao exame.

O grupo controle foi composto por 30 pacientes sem DTM, pareados por gênero, índice de maturidade esquelética vertebral cervical e classe de má oclusão. Os critérios de inclusão foram os mesmos adotados para o grupo de estudo com a exceção da necessidade da presença diagnóstica de DTM articular, Grupos II e III, de acordo com o eixo I do RDC/TMD para crianças e adolescentes - versão para a língua portuguesa ${ }^{26}$, o qual passa a ser fator de exclusão além dos demais já anteriormente relacionados para o grupo de estudo.

As telerradiografias laterais foram traçadas e os pontos cefalométricos determinados. Os pontos de referência foram digitalizados em um sistema de coordenadas X-Y através do software Radiocef (Radio Memory, Belo Horizonte, MG). As estruturas craniofaciais e suas relações foram divididas nas seguintes categorias para análise: base do crânio, maxila, mandíbula, relações intermaxilares, relações esqueléticas verticais e relações dentárias (Fig. 1A, B, C, D).

As medidas angulares e lineares foram adotadas das análises previamente descritas $7,16,20$.

As medidas encontradas foram tabuladas e a média e o desvio-padrão, para cada variável da amostra de pacientes, foram calculados com a utilização do software estatístico SPSS (SPSS Inc, Chicago).

As diferenças encontradas entre as médias, para cada uma das variáveis selecionadas, entre o grupo de pacientes com DTM articular e o grupo controle, foram avaliadas pela aplicação do teste estatístico $t$ de Student para amostras independentes. Foram consideradas estatisticamente significantes as diferenças que apresentaram uma chance menor do que $5 \%$ de terem ocorrido ao acaso $(\mathrm{p}<0,05)$.

Para testar a magnitude do erro de medição das variáveis cefalométricas no presente estudo, as telerradiografias laterais de 15 pacientes selecionados aleatoriamente foram medidas novamente. Através da utilização da fórmula de Dahlberg ${ }^{6}$ o erro situou-se entre 0,31 e $0,79 \mathrm{~mm}$ para as medidas lineares e 0,30 e 0,98 para as medidas angulares.

\section{RESULTADOS}

Os pacientes do grupo experimental - presença de DTM articular $(n=30)$ - apresentaram mediana de idade de 9 anos (limites de 5 a 17 anos), sendo 12 indivíduos (40\%) do gênero masculino e 18 (60\%) do gênero feminino. Destes, 7 estavam no estágio de maturidade vertebral cervical I (23\%), 10 no estágio II (33\%), 12 no estágio III (40\%) e 1 no estágio IV (4\%). 


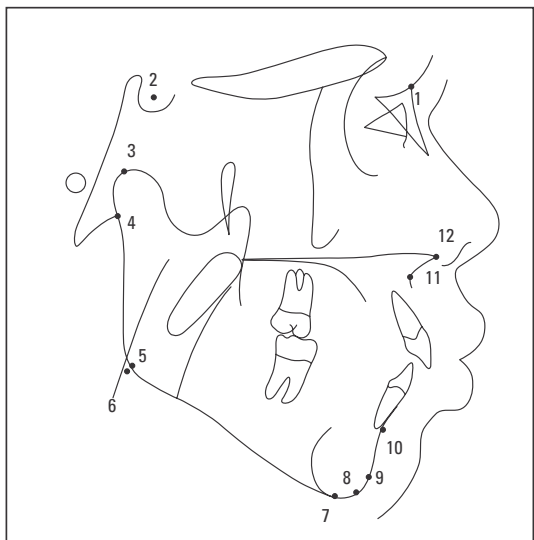

FIGURA 1A - Pontos utilizados neste estudo, 1) N; 2) S; 3) $\mathrm{Co}$; 4) $\mathrm{Ar}$; 5) Go anatômico; 6) Go cefalométrico; 7) Me; 8) $G$; 9) Pog;10) $B$; 11) $A$; 12) Ena.

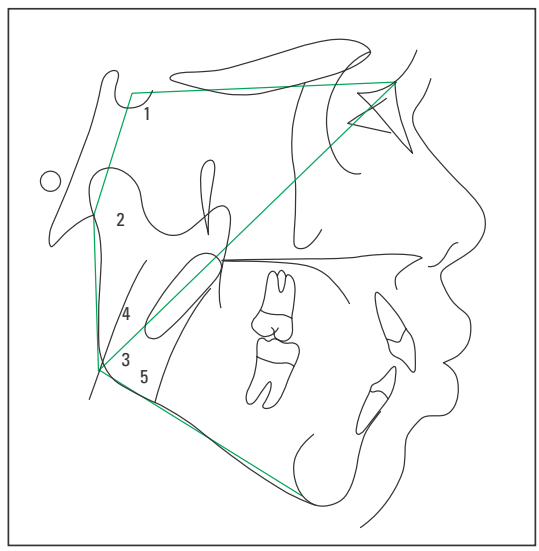

FIGURA 1C - Ângulos utilizados neste estudo, 1) S.N.Ar 2) S.Ar.Go; 3) Ar.Go.Me; 4) Ar.Go.N; 5) Na.Go.Me.

No grupo controle - ausência de DTM articular $(\mathrm{n}=30)$ - a mediana de idade foi de 10 anos (limite de 5 a 14 anos), sendo observada a mesma divisão para gênero e índice de maturidade vertebral cervical e relação intermaxilar sagital encontrada no grupo experimental em virtude do pareamento da amostra efetuado.

O grupo experimental Classe I apresentou média para os valores do ângulo sela S.N.Ar aumentada em relação à média apresentada para os valores encontrados no grupo controle, sendo estas diferenças estatisticamente significantes (Tab. 1).

O grupo experimental Classe II apresentou mé-

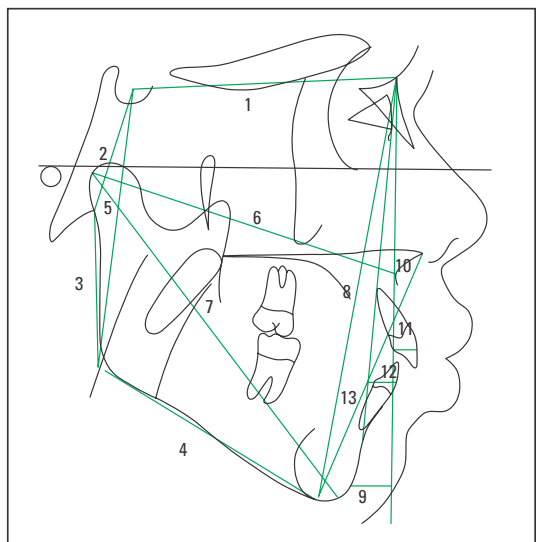

FIGURA 1B - Linhas utilizadas neste estudo, 1) S-N ; 2) S-Ar; 3) $\mathrm{Ar}-\mathrm{Go}$; 4) $\mathrm{Go}-\mathrm{Me}$; 5) $\mathrm{S}-\mathrm{Go}$; 6) $\mathrm{Co}-\mathrm{A}$; 7) $\mathrm{Co}-\mathrm{Gn}$; 8) N-Me; 9) NperpPog;10) NPerpA; 11) 1-NA; 12) 1-NB; 13) EnaMe.

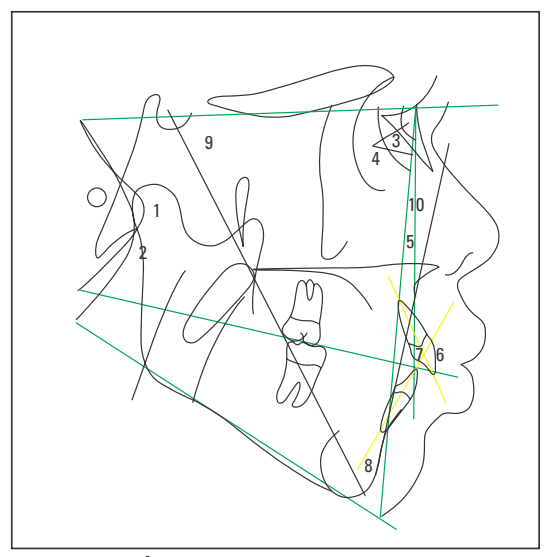

FIGURA 1D - Ângulos utilizados neste estudo (continuação), 1) SN.Po; 2) SN.GoGn; 3) S.N.A; 4) S.N.B; 5) A.N.B; 6) 1.1 ; 7) 1.NA; 8) 1.NB; 9) SN.Gn; 10) N.A.P.

dia dos valores do ângulo sela S.N.Ar e NperpA diminuídos e do ângulo articular (S.Ar.Go) aumentado em relação à média apresentada para os valores encontrados no grupo controle, tendo estas diferenças sido estatisticamente significantes (Tab. 2).

O grupo experimental Classe III apresentou média para os valores do ângulo da convexidade facial (N.A.P), inclinação dos incisivos inferiores (1.NB) e protrusão dos incisivos inferiores (1-NB) aumentados e ângulo interincisal (1.1) diminuído em relação à média apresentada para os valores encontrados no grupo controle, tendo estas diferenças sido estatisticamente significantes (Tab. 3). 
Tabela 1 - Distribuição das médias e desvio-padrão para as variáveis cefalométricas em indivíduos com relação sagital de Classe I.

\begin{tabular}{|c|c|c|c|}
\hline $\begin{array}{l}\text { Variáveis } \\
\text { cefalométricas }\end{array}$ & $\begin{array}{c}\text { Grupo } \\
\text { experimental } \\
\text { (média } \pm \text { d.p.) }\end{array}$ & $\begin{array}{c}\text { Grupo } \\
\text { controle } \\
\text { (média } \pm \text { d.p.) }\end{array}$ & $\mathbf{p}$ \\
\hline \multicolumn{4}{|l|}{ Base do crânio } \\
\hline S-N (mm) & $68,00 \pm 3,71$ & $67,83 \pm 4,58$ & 0,932 \\
\hline S-Ar (mm) & $32,36 \pm 2,62$ & $33,26 \pm 2,94$ & 0,481 \\
\hline S.N.Ar $\left({ }^{\circ}\right)$ & $126,47 \pm 5,69$ & $121,72 \pm 4,62$ & 0,050 \\
\hline \multicolumn{4}{|l|}{ Maxila } \\
\hline S.N.A $\left({ }^{\circ}\right)$ & $80,76 \pm 3,09$ & $83,24 \pm 4,51$ & 0,169 \\
\hline Co.A (mm) & $85,18 \pm 6,09$ & $86,93 \pm 3,03$ & 0,426 \\
\hline NperpA (mm) & $1,67 \pm 3,77$ & $1,44 \pm 3,31$ & 0,887 \\
\hline \multicolumn{4}{|l|}{ Mandíbula } \\
\hline S.N.B $\left({ }^{\circ}\right)$ & $76,53 \pm 2,81$ & $78,19 \pm 3,62$ & 0,266 \\
\hline Co-Gn (mm) & $111,24 \pm 5,92$ & $113,28 \pm 3,42$ & 0,358 \\
\hline NperpPog (mm) & $-4,91 \pm 3,48$ & $-5,55 \pm 3,81$ & 0,703 \\
\hline Go-Me (mm) & $70,39 \pm 4,97$ & $71,19 \pm 3,60$ & 0,687 \\
\hline \multicolumn{4}{|c|}{ Relação intermaxilar } \\
\hline A.N.B $\left({ }^{\circ}\right)$ & $4,23 \pm 1,82$ & $5,14 \pm 1,93$ & 0,292 \\
\hline N.A.P $\left({ }^{\circ}\right)$ & $8,16 \pm 5,35$ & $9,70 \pm 4,57$ & 0,498 \\
\hline \multicolumn{4}{|c|}{ Relação esquelética vertical } \\
\hline Ena-Me (mm) & $65,31 \pm 4,07$ & $66,19 \pm 3,34$ & 0,603 \\
\hline S-Go $(\mathrm{mm})$ & $70,67 \pm 6,19$ & $71,55 \pm 3,11$ & 0,693 \\
\hline $\mathrm{N}-\mathrm{Me}(\mathrm{mm})$ & $113,93 \pm 5,68$ & $112,80 \pm 5,64$ & 0,661 \\
\hline $\operatorname{Ar}-G_{0}(\mathrm{~mm})$ & $40,85 \pm 3,85$ & $40,44 \pm 2,74$ & 0,788 \\
\hline S.Ar.Go $\left({ }^{\circ}\right)$ & $144,31 \pm 7,20$ & $147,40 \pm 5,38$ & 0,291 \\
\hline Ar.Go.Me $\left({ }^{\circ}\right)$ & $125,45 \pm 8,31$ & $125,63 \pm 4,73$ & 0,955 \\
\hline SN.GoGn $\left({ }^{\circ}\right)$ & $36,39 \pm 5,90$ & $33,85 \pm 4,05$ & 0,278 \\
\hline SN.Gn $\left({ }^{\circ}\right)$ & $69,92 \pm 3,75$ & $67,53 \pm 2,16$ & 0,097 \\
\hline SN.Po $\left({ }^{\circ}\right)$ & $17,01 \pm 4,91$ & $17,64 \pm 3,89$ & 0,756 \\
\hline Ar.Go.N $\left({ }^{\circ}\right)$ & $51,52 \pm 5,21$ & $51,85 \pm 3,38$ & 0,869 \\
\hline N.Go.Me $\left({ }^{\circ}\right)$ & $73,90 \pm 5,40$ & $73,78 \pm 3,97$ & 0,954 \\
\hline Soma $\left({ }^{\circ}\right)$ & $396,28 \pm 4,97$ & $394,75 \pm 3,62$ & 0,441 \\
\hline \multicolumn{4}{|c|}{ Relações dentárias } \\
\hline 1.NA $\left({ }^{\circ}\right)$ & $25,80 \pm 6,59$ & $21,36 \pm 7,77$ & 0,185 \\
\hline 1-NA (mm) & $5,67 \pm 2,01$ & $4,83 \pm 2,95$ & 0,467 \\
\hline 1.NB $\left({ }^{\circ}\right)$ & $31,48 \pm 7,92$ & $30,17 \pm 6,70$ & 0,695 \\
\hline 1-NB (mm) & $5,02 \pm 4,25$ & $8,18 \pm 6,37$ & 0,208 \\
\hline $1.1\left(^{\circ}\right)$ & $119,11 \pm 11,57$ & $123,90 \pm 9,18$ & 0,319 \\
\hline
\end{tabular}

Tabela 2 - Distribuição das médias e desvio-padrão para as variáveis cefalométricas em indivíduos com relação sagital de Classe II.

\begin{tabular}{|c|c|c|c|}
\hline $\begin{array}{l}\text { Variáveis } \\
\text { cefalométricas }\end{array}$ & $\begin{array}{c}\text { Grupo } \\
\text { experimental } \\
\text { (média } \pm \text { d.p.) }\end{array}$ & $\begin{array}{c}\text { Grupo } \\
\text { controle } \\
\text { (média } \pm \text { d.p.) }\end{array}$ & $\mathbf{p}$ \\
\hline \multicolumn{4}{|l|}{ Base do crânio } \\
\hline S-N (mm) & $70,19 \pm 4,96$ & $68,42 \pm 5,15$ & 0,444 \\
\hline S-Ar (mm) & $34,69 \pm 3,40$ & $33,72 \pm 3,34$ & 0,529 \\
\hline S.N.Ar $\left({ }^{\circ}\right)$ & $125,01 \pm 4,20$ & $130,99 \pm 4,38$ & 0,006 \\
\hline \multicolumn{4}{|l|}{ Maxila } \\
\hline S.N.A $\left({ }^{\circ}\right)$ & $80,10 \pm 4,08$ & $81,70 \pm 3,02$ & 0,332 \\
\hline Co.A (mm) & $86,08 \pm 6,63$ & $89,58 \pm 8,20$ & 0,308 \\
\hline NperpA (mm) & $0,44 \pm 3,06$ & $3,69 \pm 3,39$ & 0,037 \\
\hline \multicolumn{4}{|l|}{ Mandíbula } \\
\hline S.N.B $\left({ }^{\circ}\right)$ & $74,53 \pm 3,76$ & $75,52 \pm 3,25$ & 0,535 \\
\hline Co-Gn (mm) & $109,73 \pm 10,85$ & $112,09 \pm 8,69$ & 0,598 \\
\hline NperpPog (mm) & $-9,05 \pm 8,60$ & $-2,71 \pm 7,88$ & 0,102 \\
\hline Go-Me (mm) & $68,01 \pm 7,14$ & $71,15 \pm 5,71$ & 0,292 \\
\hline \multicolumn{4}{|c|}{ Relação intermaxilar } \\
\hline A.N.B $\left({ }^{\circ}\right)$ & $5,57 \pm 2,69$ & $6,18 \pm 2,43$ & 0,602 \\
\hline N.A.P $\left({ }^{\circ}\right)$ & $10,43 \pm 5,90$ & $10,79 \pm 3,38$ & 0,871 \\
\hline \multicolumn{4}{|c|}{ Relação esquelética vertical } \\
\hline Ena-Me (mm) & $67,53 \pm 8,32$ & $66,49 \pm 5,81$ & 0,749 \\
\hline S-Go (mm) & $72,76 \pm 7,35$ & $71,50 \pm 5,44$ & 0,667 \\
\hline $\mathrm{N}-\mathrm{Me}(\mathrm{mm})$ & $117,26 \pm 12,08$ & $115,04 \pm 8,99$ & 0,647 \\
\hline Ar-Go (mm) & $41,33 \pm 4,29$ & $42,84 \pm 5,18$ & 0,487 \\
\hline S.Ar.Go $\left({ }^{\circ}\right)$ & $145,61 \pm 6,30$ & $139,57 \pm 6,27$ & 0,045 \\
\hline Ar.Go.Me $\left({ }^{\circ}\right)$ & $129,01 \pm 5,75$ & $125,66 \pm 4,99$ & 0,181 \\
\hline SN.GoGn $\left({ }^{\circ}\right)$ & $36,59 \pm 6,8$ & $36,02 \pm 6,26$ & 0,847 \\
\hline $\operatorname{SNGn}\left({ }^{\circ}\right)$ & $71,04 \pm 5,45$ & $70,11 \pm 4,31$ & 0,676 \\
\hline SN.Po $\left({ }^{\circ}\right)$ & $17,07 \pm 6,24$ & $15,45 \pm 4,92$ & 0,528 \\
\hline Ar.Go.N $\left({ }^{\circ}\right)$ & $52,15 \pm 5,29$ & $52,85 \pm 3,76$ & 0,739 \\
\hline N.Go.Me $\left({ }^{\circ}\right)$ & $77,01 \pm 5,28$ & $72,92 \pm 5,06$ & 0,094 \\
\hline Soma $\left({ }^{\circ}\right)$ & $399,81 \pm 6,62$ & $396,22 \pm 5,33$ & 0,198 \\
\hline \multicolumn{4}{|c|}{ Relações dentárias } \\
\hline 1.NA $\left(^{\circ}\right)$ & $23,66 \pm 3,49$ & $26,46 \pm 8,51$ & 0,349 \\
\hline 1-NA (mm) & $5,66 \pm 4,30$ & $6,26 \pm 2,27$ & 0,703 \\
\hline 1.NB $\left({ }^{\circ}\right)$ & $29,66 \pm 3,96$ & $29,27 \pm 3,36$ & 0,818 \\
\hline $1-\mathrm{NB}(\mathrm{mm})$ & $7,94 \pm 3,81$ & $6,55 \pm 2,03$ & 0,322 \\
\hline $1.1\left(^{\circ}\right)$ & $123,32 \pm 7,06$ & $118,50 \pm 10,40$ & 0,241 \\
\hline
\end{tabular}


Tabela 3 - Distribuição das médias e desvio-padrão para as variáveis cefalométricas em indivíduos com relação sagital de Classe III.

\begin{tabular}{|c|c|c|c|}
\hline $\begin{array}{l}\text { Variáveis } \\
\text { cefalométricas }\end{array}$ & $\begin{array}{c}\text { Grupo } \\
\text { experimental } \\
\text { (média } \pm \text { d.p.) }\end{array}$ & $\begin{array}{c}\text { Grupo } \\
\text { controle } \\
\text { (média } \pm \text { d.p.) }\end{array}$ & $\mathbf{p}$ \\
\hline \multicolumn{4}{|l|}{ Base do crânio } \\
\hline S-N (mm) & $68,42 \pm 2,60$ & $64,85 \pm 5,25$ & 0,070 \\
\hline $\mathrm{S}-\operatorname{Ar}(\mathrm{mm})$ & $32,08 \pm 3,01$ & $28,63 \pm 4,87$ & 0,073 \\
\hline S.N.Ar $\left({ }^{\circ}\right)$ & $121,23 \pm 6,09$ & $122,28 \pm 4,98$ & 0,680 \\
\hline \multicolumn{4}{|l|}{ Maxila } \\
\hline S.N.A $\left({ }^{\circ}\right)$ & $80,35 \pm 4,29$ & $81,20 \pm 5,54$ & 0,707 \\
\hline Co.A (mm) & $86,08 \pm 6,63$ & $89,58 \pm 8,20$ & 0,308 \\
\hline NperpA (mm) & $-0,24 \pm 4,67$ & $0,25 \pm 3,88$ & 0,800 \\
\hline \multicolumn{4}{|l|}{ Mandíbula } \\
\hline S.N.B $\left({ }^{\circ}\right)$ & $78,23 \pm 3,84$ & $80,91 \pm 4,10$ & 0,148 \\
\hline Co-Gn (mm) & $109,73 \pm 10,85$ & $112,09 \pm 8,69$ & 0,598 \\
\hline NperpPog (mm) & $-4,63 \pm 8,78$ & $0,84 \pm 4,88$ & 0,103 \\
\hline Go-Me (mm) & $70,21 \pm 6,90$ & $70,18 \pm 6,13$ & 0,991 \\
\hline \multicolumn{4}{|c|}{ Relação intermaxilar } \\
\hline A.N.B $\left({ }^{\circ}\right)$ & $5,57 \pm 2,69$ & $6,18 \pm 2,43$ & 0,602 \\
\hline N.A.P $\left({ }^{\circ}\right)$ & $4,36 \pm 3,63$ & $-0,08 \pm 5,40$ & 0,045 \\
\hline \multicolumn{4}{|c|}{ Relação esquelética vertical } \\
\hline Ena-Me (mm) & $66,02 \pm 7,77$ & $64,08 \pm 5,50$ & 0,527 \\
\hline S-Go (mm) & $68,04 \pm 6,20$ & $66,14 \pm 7,00$ & 0,528 \\
\hline $\mathrm{N}-\mathrm{Me}(\mathrm{mm})$ & $114,13 \pm 11,69$ & $109,54 \pm 8,59$ & 0,330 \\
\hline Ar-Go (mm) & $39,33 \pm 3,81$ & $39,84 \pm 3,74$ & 0,764 \\
\hline S.Ar.Go $\left({ }^{\circ}\right)$ & $146,37 \pm 7,73$ & $144,67 \pm 6,37$ & 0,597 \\
\hline Ar.Go.Me $\left({ }^{\circ}\right)$ & $129,45 \pm 4,74$ & $129,60 \pm 4,62$ & 0,943 \\
\hline SN.GoGn $\left({ }^{\circ}\right)$ & $37,58 \pm 5,91$ & $36,12 \pm 4,92$ & 0,556 \\
\hline SN.Gn $\left({ }^{\circ}\right)$ & $68,30 \pm 5,01$ & $66,67 \pm 3,24$ & 0,398 \\
\hline SN.Po $\left({ }^{\circ}\right)$ & $21,32 \pm 3,82$ & $17,51 \pm 4,72$ & 0,063 \\
\hline Ar.Go.N $\left({ }^{\circ}\right)$ & $52,82 \pm 5,62$ & $53,57 \pm 4,35$ & 0,742 \\
\hline N.Go.Me $\left({ }^{\circ}\right)$ & $76,64 \pm 4,92$ & $76,07 \pm 3,36$ & 0,766 \\
\hline Soma $\left({ }^{\circ}\right)$ & $397,05 \pm 6,62$ & $396,52 \pm 2,77$ & 0,819 \\
\hline \multicolumn{4}{|c|}{ Relações dentárias } \\
\hline 1.NA $\left({ }^{\circ}\right)$ & $27,75 \pm 6,38$ & $21,42 \pm 8,05$ & 0,067 \\
\hline 1-NA (mm) & $4,78 \pm 1,98$ & $3,83 \pm 2,19$ & 0,326 \\
\hline 1.NB $\left(^{\circ}\right)$ & $31,63 \pm 5,90$ & $20,04 \pm 6,66$ & 0,001 \\
\hline $1-\mathrm{NB}(\mathrm{mm})$ & $5,98 \pm 1,43$ & $4,10 \pm 1,75$ & 0,017 \\
\hline controle & $120,98 \pm 9,79$ & $137,64 \pm 12,44$ & 0,004 \\
\hline
\end{tabular}

\section{DISCUSSÃO}

Katzberg et al. ${ }^{17}$, em 1985 , foram uns dos primeiros autores a identificarem alterações nas medidas cefalométricas, esqueléticas e dentoalveolares, decorrentes do estabelecimento, em estágios precoces de desenvolvimento facial, de desordens da ATM, avaliadas por procedimento de artrografia.

Posteriormente, com o advento das técnicas de imagens por ressonância magnética, a morfologia da ATM pôde ser estudada de maneira não invasiva, e imagens radiográficas puderam ser correlacionadas com imagens de ressonância magnética de ambas as ATM's. Os primeiros relatos de que o desarranjo interno da ATM poderia contribuir para o desenvolvimento de retrognatia mandibular, baseados nestes estudos $^{27}$, foram motivos de grande controvérsia entre especialistas de diferentes áreas ${ }^{9}$.

Recentemente, inúmeros estudos foram publicados, utilizando IRM para o diagnóstico das alterações posicionais do disco articular e/ou degenerativas da ATM, buscando correlacionar estes diagnósticos com alterações das medidas craniofaciais de pacientes em crescimento ${ }^{12,13,14,22,23,24}$. Os resultados demonstraram, de maneira quase unânime, o estabelecimento de um padrão facial vertical e retrognata com compensações dentoalveolares compatíveis com este padrão, confirmando as proposições ${ }^{27}$.

Contudo, o custo relativamente elevado das IRM impede sua indicação rotineira na confirmação dos diagnósticos de DTM articulares, as quais são possíveis de serem detectadas clinicamente com níveis aceitáveis de validade e precisão, a qual pode chegar a 95\% de concordância nos casos de deslocamento do disco sem redução, osteoartrite e osteoartrose ${ }^{21}$.

No presente estudo, os pacientes e controles foram pareados por gênero, índice de maturidade vertebral cervical e classe de má oclusão presente. Este pareamento teve por finalidade minimizar os efeitos do padrão individual de crescimento facial nos resultados obtidos. A análise de medidas cefalométricas em pacientes adultos com desordens articulares, agrupados de acordo com a classificação da má oclusão presente, foi apenas recentemente conduzida ${ }^{15}$. 
Os resultados deste estudo demonstraram, de maneira similar ao encontrado em pacientes adultos $^{1,15}$, que crianças e adolescentes com desordens articulares e má oclusão de Classe II, quando comparados com controles com características similares, apresentam diminuição do ângulo de deflexão da base do crânio, retrusão maxilar e aumento do ângulo articular, o qual evidencia uma tendência vertical de crescimento facial. Este fato provavelmente se deve a uma diminuição do crescimento vertical do ramo mandibular, o qual processa-se no nível condilar, em ATM acometidas por deslocamentos do disco articular e/ou alterações degenerativas.

Pacientes em crescimento com má oclusão de Classe III, no presente estudo, quando comparados com controles com características similares, apresentaram aumento da convexidade facial, denotando maior retrusão mandibular, inclinação vestibular e protrusão dos incisivos inferiores, consideradas alterações dentoalveolares compensatórias às alterações esqueléticas acima relatadas. Estes resultados estão de acordo com os resultados encontrados em pacientes adultos ${ }^{15}$.

A inclinação vestibular compensatória dos incisivos inferiores está provavelmente relacionada ao aumento da sobressaliência, decorrente do posicionamento posterior da mandíbula e de sua rotação no sentido horário. Tal rotação está associada à diminuição da altura do ramo encontrada em pacientes com desordens da ATM em diferentes estudos 1,2,23,24.

As medidas do comprimento maxilar e mandibular sofrem influência direta do padrão de crescimento facial, o qual se manifesta já bastante precocemente. Em estudo de publicação recente, $\operatorname{Tesch}^{28}$, avaliando crianças em estágios precoces de desenvolvimento esquelético, demonstrou que as médias encontradas para as medidas cefalométricas relacionadas ao comprimento mandibular apresentaram aumento estatisticamente significante no grupo de pacientes com má oclusão de Classe III, quando comparados com o grupo de pacientes com má oclusão de Classe I.

As diferenças nos valores encontrados para as medidas cefalométricas de pacientes em crescimen- to com diferentes tipos de má oclusão (Classe I, II e III) e desordens da ATM, refletem, provavelmente, a influência do padrão distinto de crescimento mandibular observado nestes diferentes subgrupos diagnósticos.

Não é possivel afirmar que as alterações cefalométricas encontradas em pacientes adultos com desordens da ATM sejam conseqüência direta de modificações do crescimento decorrentes da préexistência destas desordens na infância e adolescência, pois estas também podem estar relacionadas a processos degenerativos ocorridos após o término do crescimento mandibular considerável.

Os resultados deste estudo demonstram haver relação entre desordens da ATM em crianças e adolescentes, preponderantemente com má oclusão de Classe II e III, e a presença de um padrão hiperdivergente de crescimento facial, contudo o sentido desta associação não pôde ser determinado por tratar-se de um estudo transversal, onde os supostos fatores de risco e desfecho foram avaliados em um mesmo momento. Portanto, a hipótese destas desordens influenciarem o padrão de crescimento facial precisa ser confirmada através do acompanhamento longitudinal, até a fase adulta, de um grupo de pacientes em crescimento, portadores ou não de desordens da ATM.

Caso esta hipótese possa ser comprovada, maior atenção deveria ser dispendida à identificação precoce de sinais clínicos de desordens da ATM em estágios iniciais de desenvolvimento, principalmente em pacientes com má oclusão de Classe II ou III, as quais poderiam ser mais adequadamente diagnosticadas e mensuradas por técnicas de imagem, como a IRM $^{5}$. Desta forma, protocolos terapêuticos adequados poderiam ser instituídos a fim de evitar ou minimizar as influências indesejáveis destas desordens no padrão de crescimento facial.

\section{CONCLUSÕES}

De acordo com os resultados do presente estudo pode-se concluir que foram encontradas diferenças entre variáveis cefalométricas de crianças com DTM 
articular e grupo controle livre da referida desordem, evidenciando uma padrão vertical de crescimento mandibular; tendo estas diferenças sido mais expressivas nos pacientes que apresentam desproporção entre o crescimento intermaxilar (Classe II e III).

Enviado em: abril de 2007

Revisado e aceito: novembro de 2007

\title{
Cephalometric changes present in children and adolescents with TMJ disorders with different classes of malocclusions
}

\begin{abstract}
Introduction: the involvement of TMJ through degenerative changes and displacements of the articular disc proved to be an important risk factor when identifying individuals with vertical facial growth patterns. Purposes: to evaluate differences between cephalometric variables in children with articular TMD and a control group, checking the variations in relation to the different patterns of malocclusion (Class I, II and III). Method: the study included children and adolescents of up to a maximum age of 17 , who were divided up into an experimental group $(n=30)$ diagnosed with articular TMD, diagnosed in accordance with the RDC/TMD axis I for children and adolescents, and a control group $(n=30)$ without $T M D$, further paired by gender, stage of cervical vertebral skeletal maturity and class of malocclusion. Lateral cephalogram was carried out and both the craniofacial structures and their relations were separated into: the cranial base, maxillary, mandible, intermaxillary relations, vertical skeletal relations and dental relations. The differences found between the means, for each of the variables, were evaluated through the application of the Student t statistical test for independent samples. Results: the Class I experimental group presented increased S.N.Ar $(p=0.05)$. The Class II experimental group presented reduced S.N.Ar $(p=0.006)$ and NperpA $(p=0.037)$ and increased S.Ar.Go $(p=0.045)$. The Class III experimental group presented increased N.A.P $(p=0.045)$, 1.NB $(p=0.001)$ and $1-N B(p=0.017)$ and reduced $1.1(p=0.004)$. Conclusion: differences were more significant in those patients who presented Class II and III malocclusions.
\end{abstract}

Key words: Skeletal pattern. TMD. Malocclusion.

\section{REFERÊNCIAS}

1. AHN S, J. KIM, T. W. NAHM, D S. Cephalometrric keys to internal derangement of temporomandibular joint in women with Class II maloclusion. Am. J. Orthod. Dentofacial Orthop., St. Louis, v. 126, p. 486-495, 2004

2. AHN, S. J.BAEK, S. H. KIM, T. W. NAHM, D. S. Discrimination of internal derangement of temporomandibular joint by lateral cephalometric analysis. Am. J. Orthod. Dentofacial Orthop. St. Louis, v. 130, no. 3, p. 331-339, Sept. 2006

3. BRAND, J. W.; NIELSON, K. J.; TALLENTS, R. H.; NANDA, R. S.; CURRIER, G. F.; OWEN, W. L. Lateral cephalometric analysis of skeletal patterns in patients with and without internal derangement of the temporomandibular joint. Am. J. Orthod. Dentofacial Orthop., St. Louis, v. 107, no. 2, p. 121-128, Feb. 1995
4. BYUN, E S : AHN S. J : KIM , T W R Relationship between internal derangement of the temporomandibular joint and dentofacial morphology in women with anterior open bite. Am. J. Orthod. Dentofacial Orthop., St. Louis, v. 128, no. 1 p. 87-95, July 2005

5. BENBELAID, R.; FLEITER, B.; ZOUAOUI, A.; GAUDY, J. F. Proposed graphical system of evaluating disc-condyle displacements of the temporomandibular joint in MRI. Surg. Radiol. Anat., Berlin, v. 27, no. 5, p. 361-367, Dec. 2005

6. DAHLBERG, G. Statistical methods for medical and biological students. New York: Interscience, 1940.

7. DOWNS, W. B. Variation in facial relationship: their significance in treatment and prognosis. Am. J. Orthod., St. Louis, v. 34 p. $812-840,1948$ 
8. DWORKIN, S. F.; LERESCHE, L. Research diagnostic criteria for temporomandibular disorders: review, criteria, examinations and specifications. Critique. J. Craniomandib. Disord. Lombard, v. 6, p. 302, 1992.

9. DRANGSHOLT, M. T.; LERESCHE, L.; RAMSEY, D. S.; HERRING S.; DWORKIN, S. F. Comments on pediatric internal derangements. Am. J. Orthod. Dentofacial Orthop., St. Louis, v. 104, no. 1, p. 51-59, July 1993.

10. EGEMMARK-ERIKSSON, I. CARLSSON, G. E. INGERVALL, B. Prevalence of mandibular dysfunction and orofacial parafunction in 7, 11 and 15-year-old Swedish children. Eur. J. Orthod. Oxford, v. 3, p. 163-72, 1981

11. GOLDSTEIN, B. H. Temporomandibular disorders: a review of current understanding. Oral Surg. Oral Med. Oral Pathol. Oral Radiol. Endod., St. Louis, v. 88, p. 379-385, 1999

12. GIDARAKOU, I. K.; TALLENTS, R. H.; KYRKANIDES, S.; STEIN, S.; MOSS, M. E. Comparison of skeletal and dental morphology in asymptomatic volunteers and symptomatic patients with bilateral disk displacement with reduction. Angle Orthod. Appleton, v. 72, p. 541-546, 2002.

13. GIDARAKOU, I. K.; TALLENTS, R. H.; KYRKANIDES, S.; STEIN, S.; MOSS, M. E. Comparison of skeletal and dental morphology in asymptomatic volunteers and symptomatic patients with bilateral degenerative joint disease. Angle Orthod., Appleton, v. 73, p. 71-78, 2003.

14. GIDARAKOU, I. K.; TALLENTS, R. H.; KYRKANIDES, S.; STEIN, S.; MOSS, M. E. Comparison of skeletal and dental morphology in asymptomatic volunteers and symptomatic patients with normal temporomandibular joints. Angle Orthod., Appleton, v. 73, p. $116-120,2003$

15. HWANG, C. J.; SUNG, S. J.; KIM, S. J. Lateral cephalometric characteristics of malocclusion patients with temporomandibular joint disorder symptoms. Am. J. Orthod. Dentofacial Orthop. St. Louis, v. 129, no. 4, p. 497-503, Apr. 2006

16. JARABAK, J. R.; FIZZEL, J. A. Technique and treatment with light wire appliances. St. Louis: C. V. Mosby, 1972. v. 1, 2.

17. KATZBERG, R. W.; TALLENTS, R. H.; HAYAKAWA, K.; MILLER T. L.; GOSKE, M. J.; WOOD, B. P. Internal derangements of the temporomandibular joint: findings in the pediatric age groups. Radiology, Easton, v. 154, p. 125, 1985.

18. LERESCHE, L. Epidemiology of temporomandibular disorders: implications for the investigation of etiologic factors. Crit. Rev. Oral Biol. Med., Alexandria, v. 8, p. 291-305, 1997.

19. LIST, T.; WAHLUND, K.; WENNEBERG, B.; DWORKIN, S. F. TMD in children and adolescents: prevalence of pain, gender differences, and perceived treatment need. J. Orofac. Pain, Carol Stream, v. 13, p. 9-20, 1999.

20. McNAMARA JR., J. A.; HOWE, R. P.; DISCHINGER, T. G. A comparison of Herbst and Frankel treatment in Class II malocclusion. Am. J. Orthod. Dentofacial Orthop., St. Louis, v. 98, p. $134-144,1990$

21. JOHN, M. T.; DWORKIN, S. F.; MANCL, L. A. Reliability of clinical temporomandibular disorder diagnoses. Pain, Amsterdam, v. 118, n. $1 / 2$, p. 61-69, Nov. 2005

22. NEBBE, B.; MAJOR, P. W.; PRASAD, N. G. Adolescent female craniofacial morphology associated with advanced bilateral TMJ disk displacement. Eur. J. Orthod., Oxford, v. 20, p. 701-712, 1998

23. NEBBE, B.; MAJOR, P. W.; PRASAD, N. G. Female adolescent facial pattern associated with TMJ disk displacement and reduction in disk length: part I. Am. J. Orthod. Dentofacial Orthop., St. Louis, v. 116, p. 168-178, 1999.

24. NEBBE, B.; MAJOR, P. W.; PRASAD, N. G. Male adolescent facial pattern associated with TMJ disk displacement and reduction in disk length: part II. Am. J. Orthod. Dentofacial Orthop., St. Louis, v. 116, p. 301-307, 1999

25. NILNER, M.; LASSING, S. Prevalence of functional disturbances and diseases of the stomatognathic system in 7-14 year old Swed. Dent. J., Jönköping, v. 5, p. 173-187, 1981.

26. PEREIRA, M. G. M.; TESCH, R. S.; DENARDIN, O. V. P.; FAVILLA, E. E. Versão para a língua portuguesa do questionário das características da dor, suas conseqüências e sintomas associados com Disfunção Temporomandibular em criança e adolescentes e do exame de suas condições físicas segundo o RDC/TMD. . Rev . Bahiana de Saúde Pública, Salvador, v. 31, n. 2, p. 201-211, jul./dez. 2007.

27. SCHELLHAS, K. P.; POLLEI, S. R.; WILKES, C. H. Pediatric internal derangements of the temporomandibular joint: effect on facial development. Am. J. Orthod. Dentofacial Orthop. St. Louis, v. 104, v. 1, p. 51-59, 1993.

28. TESCH, R. Morphologic characteristics of Brazilian children with class III malocclusion. ANNUAL CONGRESS AMERICAN ASSOCIATION OF ORTHODONTICS AND DENTOFACIAL ORTHOPEDICS, 105., 2005. Abstract...[S.I.:s.n.], 2005.

29. WAHLUND, K.; LIST, T.; DWORKING, S. F. Temporomandibular disorders in children and adolescents: reliability of a questionnaire, clinical examination, and diagnosis. J. Orofac. Pain, Carol Stream, v. 12, no. 12, p. 42-51, 1998.

30. WAHLUND, K.; LIST, T.; LARSON, B. Treatment of temporomandibular disorders among adolescents: a comparison between occlusal appliance, relaxation training and brief information. Acta Odontol. Scand., Oslo, v. 61, p. 203-211, 2003
Endereço para correspondência

Leticia Vilaça Willeman Bastos

Rua Ajuratuba 121 bloco A / 403 - Méier

CEP: 20.735-050 - Rio de Janeiro / RJ

E-mail: leticiawilleman@ig.com.br 